\title{
Photonic Bandgap Microcavities With Flat-Top Response
}

\author{
Chyong-Hua Chen and Yeshaiahu Fainman, Fellow, IEEE
}

\begin{abstract}
In this paper, the analysis and the design of a photonic bandgap (PBG) microcavity waveguide filter with flat-top spectral characteristics are theoretically presented. The split filter analysis and the bidirectional eigenmode propagation method are applied to the investigation of this waveguide structure to obtain the design parameters of the filter with nearly unity transmittance and a squared passband. It shows that the radiation loss in the PBG mirrors diminishes the transmittance of the filter, and a mirror with anomalous dispersion region in reflection phase could be used to assemble an optical filter with the flat-top passband. A 1-D PBG monorail waveguide microcavity filter centered at $1554 \mathrm{~nm}$ with a maximum transmission of $-0.55 \mathrm{~dB}$ and full-width at halfmaximum (FWHM) of $13.6 \mathrm{~nm}$ is numerically carried out by using 3-D finite-difference time-domain method.
\end{abstract}

Index Terms-Fabry-Pérot (FP) resonators, integrated optics, photonic bandgap (PBG), photonic-crystal (PC) waveguide, photonic integrated circuits (PICs), optical bandpass filter, optical components, optical waveguide filter, periodic structures, silicon photonics.

\section{INTRODUCTION}

W AVEGUIDE-BASED integrated optics in silicon is a promising platform for the implementation of photonic integrated circuits (PICs) by use of well-established CMOS fabrication techniques and a monolithic integration of optical and electronic devices on the same substrates [1]-[7]. The highindex contrast between silicon and cladding materials (e.g., air) enables extremely compact waveguides with very strong mode field confinement, and these waveguide components associated with photonic bandgap (PBG) structures achieve wide bandwidths [8]-[15], thereby providing good prospects for applications in optical wavelength-division multiplexing (WDM) systems.

Of the applications in optical WDM, a compact optical filter having flat-top and unity passband bandwidth, sharp rolloff on band edges, and sidelobe-free stopbands are desired in order to ensure signal fidelity and to enhance the tolerance of signal wavelength shifts. A variety of optical filters with nearly squared passband has been developed, such as thin-film filters [16], [17], diffraction grating arrays [18], waveguide gratings (AWGs) [19], unbalanced Mach-Zehnder (MZ) interferometers [20]-[22], and photonic bandgap (PBG) microcavity

Manuscript received September 7, 2006; revised February 1, 2007.

C.-H. Chen is with the Department of Photonics and Institute of ElectroOptical Engineering, National Chiao Tung University, Hsinchu 30010, Taiwan, R.O.C. (e-mail: chyong@mail.edu.tw).

Y. Fainman is with the Department of Electrical and Computer Engineering, University of California at San Diego, La Jolla, CA 92093-0407 USA (e-mail: fainman@ece.ucsd.edu).

Digital Object Identifier 10.1109/JSTQE.2007.893079 waveguides. However, thin film-filters and diffraction gratings are difficult to integrate with other passive or active optical devices in PICs. AWGs have complex structures and are not flexible to flat-top passband tailoring as a consequence of the increase in insertion loss up to several decibels. An unbalanced MZ interferometer requires several stages to achieve a flat-top spectral response, and thus, the complexity and size increase drastically with the number of the stages.

On the other hand, PBG microcavity waveguides are compact and compatible in integration. A diversity of optical PBG microcavities has been developed such as 1-D photonic crystal (PC) mirrors with a defect [8]-[15]. However, most of them were demonstrated by inserting a small defect length between two PBG mirrors as a result of a Lorentzian filter response of a Fabry-Pérot (FP) cavity, which are inappropriate for the WDM application. The investigations on flat-top microcavity waveguides were conducted by applying direct-coupled resonator design techniques [23], [24] or fiber Bragg grating concepts [25], [26] to appropriately choose microcavity sizes and reflectances of PBG mirrors in a cascaded multiple-cavity waveguide structure. However, the effect of the radiation loss embedded in the PBG structure due to a mode mismatch was not taken into account in these designs. In addition, the performances of these designed filters were numerically calculated using 2-D geometrical structures.

In this paper, we present an analytical and systematic approach to design the bandpass microcavity filter with approximately unity transmittance and nearly squared passband. We examine the relation between the radiation loss and transmittance of a PBG microcavity structure and find that the transmission properties of such a microcavity filter is approximately the same as that of an FP resonator as the radiation loss is minimized. It is known that an FP resonator with a flat-top passband could be achieved by using anomalous dispersion (AD) mirrors in the thin-film filter design techniques [27], [28]. Accordingly, we describe the design procedures to assemble an $\mathrm{AD}$ mirror by using PBG microcavities and to build a nearly squared bandpass filter consisting of two identically designed AD mirrors and appropriately choosing the separation length between these mirrors in order to compensate the phase dispersion caused by these mirrors.

The organization of this paper is as follows. In Section II, we analyze a generalized PBG microcavity waveguide by using the split filter analysis [29]-[31], obtain a formula to describe the transmission properties of this waveguide filter by the bidirectional eigenmode propagation methods [32]-[34], and discuss the effect of the radiation loss on the design parameters of the waveguide filter. As a result of the aforementioned investigation, 




Fig. 1. Schematic of a generalized PBG microcavity waveguide structure.

in Section III, we describe the design procedures and demonstrate its application for achieving a 1-D PBG monorail waveguide microcavity filter with high transmission and square shape in the passband. In addition, the performance of this filter is numerically simulated by using 3-D finite-difference time-domain (FDTD) method and compared to the theoretical calculation by using the formula obtained in Section II. In Section IV, we conclude this paper.

\section{Analysis of PBG WaVeguide Microcavities}

The diagram to represent a generalized PBG microcavity waveguide with multiple cavities is shown in Fig. 1. The whole structure is divided into two systems, system $\mathrm{A}_{1}$ and system $\mathrm{A}_{2}$, separated by the cavity $\mathrm{C}$ with the length $d$. Let the optical eigenmode fields $\left\{{ }^{\mathrm{i}} E_{\mathrm{gF}},{ }^{\mathrm{i}} E_{\mathrm{rF}},{ }^{\mathrm{i}} E_{\mathrm{gB}},{ }^{\mathrm{i}} E_{\mathrm{rB}}\right\}$, and $\left\{{ }^{\circ} E_{\mathrm{gF}},{ }^{\circ} E_{\mathrm{rF}},{ }^{\circ} E_{\mathrm{gB}},{ }^{\circ} E_{\mathrm{rB}}\right\}$ denote the forward $(\mathrm{F})$ and backward (B) waves for guided (g) and radiation (r) modes in the input and output regions, respectively. Also, $\left\{{ }^{\mathrm{a}} E_{\mathrm{gF}},{ }^{\mathrm{a}} E_{\mathrm{rF}},{ }^{\mathrm{a}} E_{\mathrm{gB}},{ }^{\mathrm{a}} E_{\mathrm{rB}}\right\}$ describe the traveling waves at the interface between the cavity $\mathrm{C}$ and system $\mathrm{A}_{1}$, whereas $\left\{{ }^{\mathrm{a}} E_{\mathrm{gF}},{ }^{a} E_{\mathrm{rF}},{ }^{a} E_{\mathrm{gB}},{ }^{a \prime} E_{\mathrm{rB}}\right\}$ represent the traveling waves at the interface between the cavity $\mathrm{C}$ and system $\mathrm{A}_{2}$. The relations between $\left\{{ }^{\mathrm{i}} E_{\mathrm{gF}},{ }^{\mathrm{i}} E_{\mathrm{rF}},{ }^{\mathrm{i}} E_{\mathrm{gB}},{ }^{\mathrm{i}} E_{\mathrm{rB}}\right\},\left\{{ }^{\mathrm{a}} E_{\mathrm{gF}},{ }^{\mathrm{a}} E_{\mathrm{rF}},{ }^{\mathrm{a}} E_{\mathrm{gB}},{ }^{\mathrm{a}} E_{\mathrm{rB}}\right\}$, and $\left\{{ }^{a} E_{\mathrm{gF}},{ }^{\mathrm{a}} E_{\mathrm{rF}},{ }^{a} E_{\mathrm{gB}},{ }^{a} E_{\mathrm{rB}}\right\},\left\{{ }^{\circ} E_{\mathrm{gF}},{ }^{\circ} E_{\mathrm{rF}},{ }^{\circ} E_{\mathrm{gB}},{ }^{\circ} E_{\mathrm{rB}}\right\}$ can be expressed in the form of the following matrix equations analyzed by using the bidirectional eigenmode propagation methods [32]-[34]:

$$
\begin{aligned}
& {\left[\begin{array}{l}
{ }^{\mathrm{i}} E_{\mathrm{gF}} \\
{ }^{\mathrm{i}} E_{\mathrm{rF}} \\
{ }^{\mathrm{i}} E_{\mathrm{gB}} \\
{ }^{\mathrm{i}} E_{\mathrm{rB}}
\end{array}\right]=\left[\begin{array}{llll}
p_{11} & p_{12} & p_{13} & p_{14} \\
p_{21} & p_{22} & p_{23} & p_{24} \\
p_{31} & p_{32} & p_{33} & p_{34} \\
p_{41} & p_{42} & p_{34} & p_{44}
\end{array}\right]\left[\begin{array}{l}
{ }^{\mathrm{a}} E_{\mathrm{gF}} \\
{ }^{\mathrm{a}} E_{\mathrm{rF}} \\
{ }^{\mathrm{a}} E_{\mathrm{gB}} \\
{ }^{\mathrm{a}} E_{\mathrm{rB}}
\end{array}\right]} \\
& {\left[\begin{array}{c}
\mathrm{a}^{\prime} E_{\mathrm{gF}} \\
{ }^{\mathrm{a}^{\prime}} E_{\mathrm{rF}} \\
{ }^{\mathrm{a}^{\prime}} E_{\mathrm{gB}} \\
{ }^{\prime} E_{\mathrm{rB}}
\end{array}\right]=\left[\begin{array}{llll}
q_{11} & q_{12} & q_{13} & q_{14} \\
q_{21} & q_{22} & q_{23} & q_{24} \\
q_{31} & q_{32} & q_{33} & q_{34} \\
q_{41} & q_{42} & q_{34} & q_{44}
\end{array}\right]\left[\begin{array}{c}
{ }^{\mathrm{o}} E_{\mathrm{gF}} \\
{ }^{\mathrm{o}} E_{\mathrm{rF}} \\
{ }^{\circ} E_{\mathrm{gB}} \\
{ }^{\circ} E_{\mathrm{rB}}
\end{array}\right]} \\
& {\left[\begin{array}{c}
{ }^{\mathrm{a}} E_{\mathrm{gF}} \\
{ }^{\mathrm{a}} E_{\mathrm{rF}} \\
{ }^{\mathrm{a}} E_{\mathrm{gB}} \\
{ }^{\mathrm{a}} E_{\mathrm{rB}}
\end{array}\right]=\left[\begin{array}{cccc}
e^{j \beta_{g} d} & 0 & 0 & 0 \\
0 & e^{j \beta_{r} d} & 0 & 0 \\
0 & 0 & e^{-j \beta_{g} d} & 0 \\
0 & 0 & 0 & e^{-j \beta_{r} d}
\end{array}\right]\left[\begin{array}{c}
\mathrm{a}^{\prime} E_{\mathrm{gF}} \\
\mathrm{a}^{\prime} E_{\mathrm{rF}} \\
{ }^{\mathrm{a}^{\prime}} E_{\mathrm{gB}} \\
{ }^{\mathrm{a}^{\prime}} E_{\mathrm{rB}}
\end{array}\right]}
\end{aligned}
$$

where $p$ and $q$ are transfer elements of the modes for system $\mathrm{A}_{1}$ and system $\mathrm{A}_{2}$, respectively. Here $\beta_{g}$ and $\beta_{r}$ are the corresponding propagation constants for guided and radiation modes in the waveguide of the cavity $\mathrm{C}$.

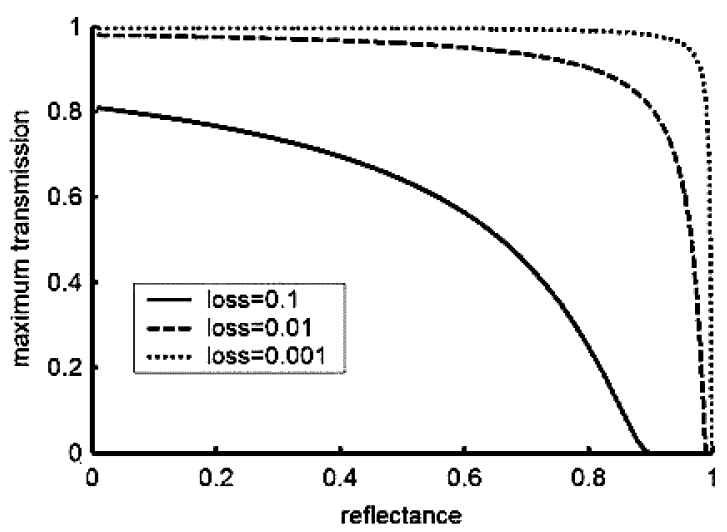

Fig. 2. Maximum transmission of an FP resonator as a function of the reflectance of the mirror with the loss of the mirror equal to 0.1 (solid line), 0.01 (dashed line), and 0.001 (dotted line).

Suppose that only one guided mode exists in the input waveguide, cavity $\mathrm{C}$, and output waveguide, and the structure itself is mirror image symmetric to the center of the cavity $\mathrm{C}$. The transmitted ratio of the guided wave obtained in the output medium to the input guided wave is denoted as

$$
\frac{1}{p_{11} p_{33} e^{j \beta_{g} d} 1+\left(p_{31}^{2} / p_{11} p_{33} e^{-2 j \beta_{g} d}\right)+h\left(p_{12}, p_{14}, p_{21}, p_{22} \ldots\right)}
$$

where $h\left(p_{12}, p_{14}, p_{21}, \ldots\right)$ is a function relevant to the coupling ratio between the guided and radiation modes via the perturbations of the structures. By examining (4), we find this formula will be identical to the transmission expression of an FP resonator if $h\left(p_{12}, p_{14}, p_{21}, \ldots\right)=0$.

To accomplish high performance of an optical bandpass filter, two main issues are needed to be addressed in the subsequent subsection: 1) high transmission in the passband and 2) flat-top passband.

\section{A. Maximum Transmission}

As seen from (4), the maximum transmission might be less than 1 due to the interactions between the radiation mode of the waveguide and the Bloch wave of the PC mirror, i.e., $h\left(p_{12}, p_{14}, p_{21}, \ldots\right) \neq 0$. The maximum transmission $\left(T_{\max }\right)$ can be expressed simply as a formula of $T^{2}(1-R)^{-2}$, where $R$ and $T$ are reflectance and transmittance of the mirror, respectively. The effect of interaction terms is included in the reflectance $R$. In Fig. 2, we show the variation of $T_{\max }$ with different values of $R$ and the loss of the mirrors. The loss $(L)$ is defined as $1-R-T$. We see that $T_{\max }$ decreases as the reflectance $R$ increases and dramatically approaches 0 as $R$ approaches $1-L$. Additionally, $T_{\max }$ increases with the decrease in the loss $L$.

Let us define a parameter $\kappa=L(1-R)^{-1}$ to describe the effect of the loss on the maximum transmission. $T_{\max }$ is simplified as an expression of $(1-\kappa)^{2}$, a parabolic function of $\kappa$. As $\kappa \rightarrow 0$, i.e., loss $=0$ and $T_{\max }=1$. On the contrary, as $\kappa \rightarrow 1$, $T_{\max }=0$. In addition, with a fixed $\kappa$, i.e., $T_{\max }$ is fixed, the larger the reflectance $R$ is, the smaller is the loss $L$ that is needed. 


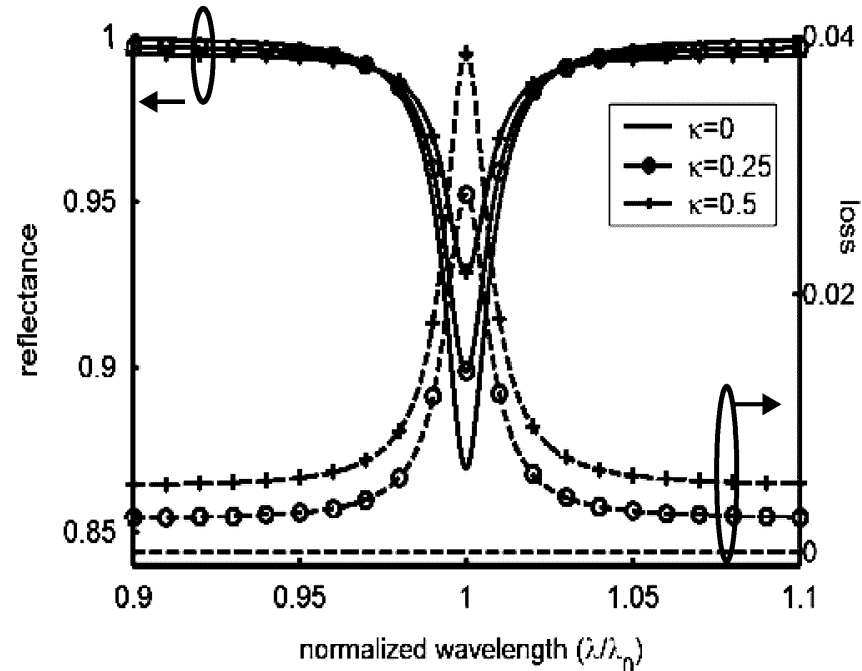

(a)

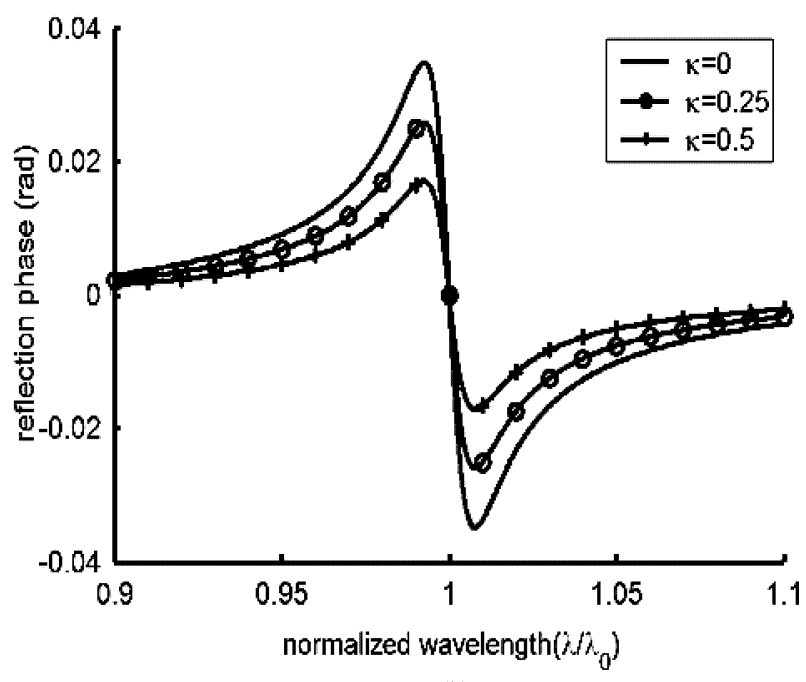

(b)

Fig. 3. (a) Reflectance (solid line) and the loss (dashed line) spectra and (b) the reflection phase spectra of the constructed AD mirrors with $\kappa=0$ (no symbol), $\kappa=0.5$ (symbol "o"), and $\kappa=0.8$ (symbol "+") of both input and output mirrors.

For example, to obtain $T_{\max }$ larger than 0.9 (or $-0.5 \mathrm{~dB}$ ), i.e., $\kappa<0.051, L$ has to be smaller than $5 \times 10^{-4}$ as $R=0.99$. However, $L$ has only to be smaller than 0.07 when $R$ becomes 0.86 . To summarize, high transmission could be achieved as $\kappa \rightarrow 0$, and this could be realized by reducing the losses of the mirrors, and preferably by using smaller reflectances of the mirrors.

\section{B. Flat-Top Passband}

In Section II-A, we obtained the condition that losses of the mirrors should be small to achieve high transmission in the passband. It means that most of the power of the guided mode is converted into that of the Bloch wave in the mirror, and vice versa. In this way, the perturbation of interaction terms could be neglected, i.e., $h\left(p_{12}, p_{14}, p_{21}, \ldots\right) \approx 0$. Therefore, (4) could approximately be described as

$$
\frac{1}{p_{11} p_{33} e^{j \beta_{g} d}\left(1+p_{31}^{2} / p_{11} p_{33} e^{-2 j \beta_{g} d}\right)} .
$$

Let $\sqrt{p_{11} p_{33}}=t e^{j \phi_{t}}$ and $\sqrt{p_{31}^{2} / p_{11} p_{33}}=r e^{j\left(\phi_{A}+\pi / 2\right)}$, where $t, r, \phi_{t}$, and $\phi_{A}$ are real numbers, representing the amplitudes and phases of the transmitted and reflected fundamental guided waves in the input (or output) waveguide and in the cavity $\mathrm{C}$. The transmittance of the filter can be calculated by

$$
\frac{t^{4}}{\left(1+r^{4}+2 r^{2} \cos \left(2 \beta_{g} d-2 \phi_{A}\right)\right)}
$$

Equation (6) is an expression of an FP resonator except that the radiation loss is included, i.e., $1-r^{2}-t^{2} \neq 0$. Based on the principles of thin-film bandpass filter designs, an FP resonator with flat-top and high transmission passband is achieved by satisfying the following conditions [27], [28]:

$$
\begin{gathered}
\Phi=2 \beta_{g} d-2 \phi_{A}=2 m \pi \\
\frac{d \Phi\left(\lambda_{0}\right)}{d \lambda} \approx 0
\end{gathered}
$$

where $\Phi$ is the total phase shift occurred inside the cavity $\mathrm{C}, m$ is an integer, and $\lambda_{0}$ is the center wavelength.

For satisfying (8), we need a specific mirror possessing AD region in its reflection phase is required (called an $\mathrm{AD}$ mirror), which could be carried out by the use of a multiple-cavity structure. In addition, an AD mirror with a one-cavity structure is obtained, as the reflectance of the mirror next to the input medium (called input mirror) is larger than that next to the output medium (called output mirror) [35], [36]. The AD region in reflection phase of the $\mathrm{AD}$ mirror is obtained at the wavelength where the local minimal reflectance has occurred, called the resonant wavelength, i.e., the total phase shift inside the cavity is $2 \pi m$, where $m$ is an integer. The resonant wavelength has to be the same as the center wavelength of the bandpass filter built by this $\mathrm{AD}$ mirror.

Next, we discuss the effect of the loss of the input and output mirrors on the performances of the constructed AD mirror and of the assembled filter. Fig. 3(a) shows the effect of $\kappa$ for both input and output mirrors on the reflectance and the loss of an AD mirror constructed by these mirrors with their reflectances of 0.99 and 0.75 , respectively. Here, we assume that the reflectances and losses of these mirrors are independent of the wavelength. We observe that the reflectance of the constructed AD mirror increases around the center wavelength as $\kappa$ increases. The reflectance due to the increase in $\kappa$ varies dramatically at the center wavelength. In addition, the maximum loss is also obtained at the center wavelength. In Fig. 3(b), we observe the AD region in reflection phase around the center wavelength. In addition, the slope of reflectance phase around this spectrum decreases as $\kappa$ increases.

Using two identical AD mirrors to build a flat-top bandpass filter separated by a cavity length $d$ for satisfying (7) and (8), we calculate the transmittance spectrum and the total phase shift $\Phi$ of this filter by using formulas of (6) and (7). The results are shown in Fig. 4(a) and (b). Fig. 4(a) illuminates that 


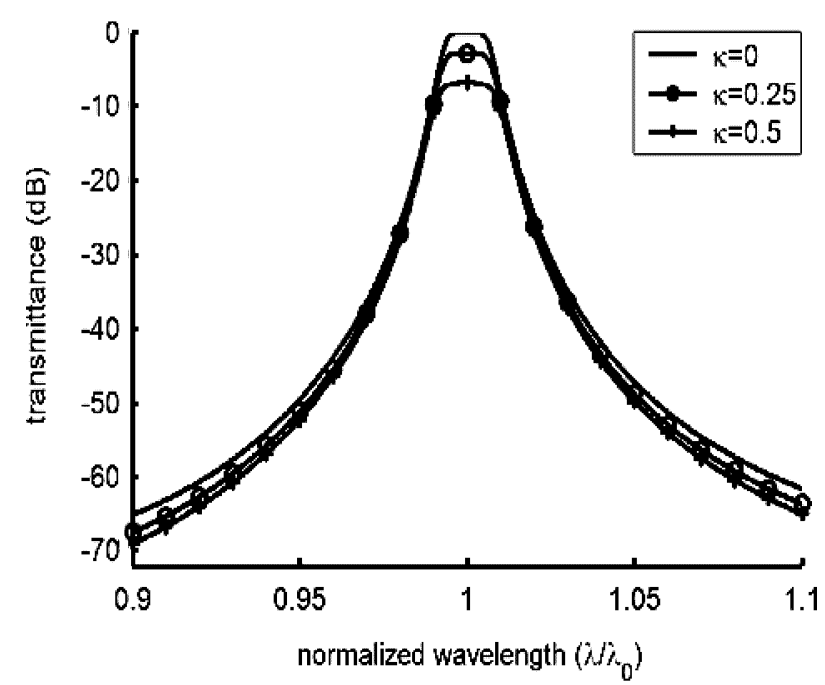

(a)

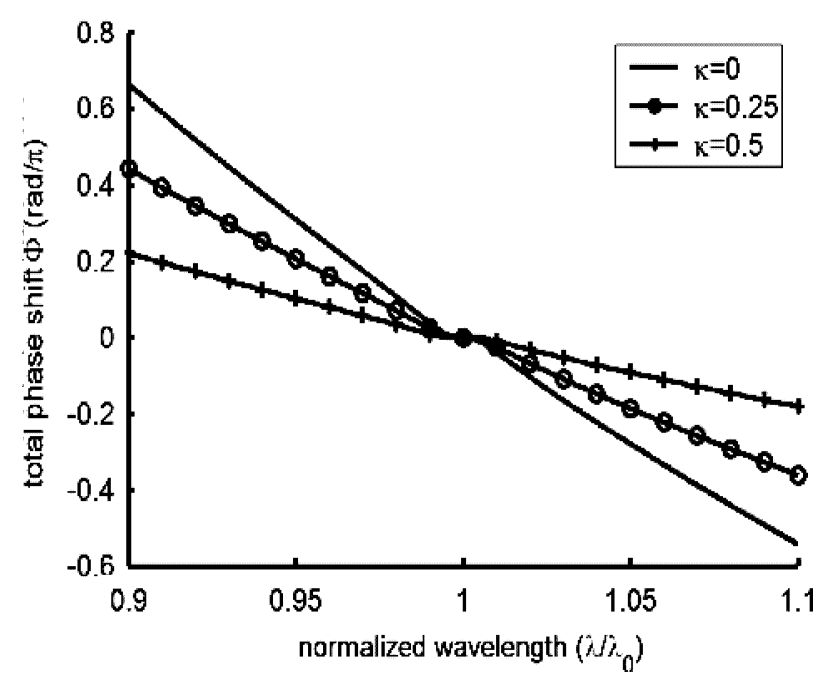

(b)

Fig. 4. (a) Transmission spectra and (b) the corresponding total phase shift $\Phi$ inside the cavity $\mathrm{C}$ of an FP resonator consisting of two identical one-cavity $\mathrm{AD}$ mirrors in the cases of $\kappa=0$ (solid line), $\kappa=0.25$ (solid line + circle), and $\kappa=0.5$ (solid line + plus symbol) for both input and output mirrors.

flat passband responses are obtained at different $\kappa$ values, but the maximum transmittance becomes smaller as $\kappa$ increases. In Fig. 4(b), we observe that around the center wavelength the total phase shift $\Phi$ is approximately constant and equal to $2 \pi m$, corresponding to (7) and (8). In Fig. 3(b), it is seen that the slope of reflectance phase is smaller as $\kappa$ increases, and thus, in order to match the condition of (8), the cavity length $d$ needs to be smaller. Consequently, the slope of the total phase shift $\Phi$ outside the flat region is much steeper as $\kappa$ approaches 0 .

In summary, a flat-top bandpass filter could be obtained by assembling two identical AD mirrors separated by a cavity with its length $d$ satisfying (7) and (8). In addition, the values of $\kappa$ for both input and output mirrors affect the shape of the built filter a little, but strongly the maximum transmittance in the passband. The larger the value of $\kappa$ the larger is the reflectance, the larger the loss, and less steeper is the slope of the reflection phase of the constructed $\mathrm{AD}$ mirror at the center wavelength.

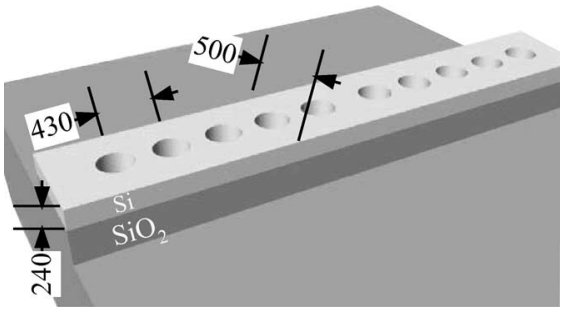

Fig. 5. Schematic structure of PBG monorail waveguide. Unit of each label is in nanometers.

Consequently, larger $\kappa$ of the $\mathrm{AD}$ mirror and smaller maximum transmittance are realized.

\section{IMPLEMENTATION OF A BANDPASS WAVEGUIDE FILTER WITH SQUARED PASSBAND}

In this section, we explore a PBG waveguide microcavity to demonstrate our approach. A high lateral index-contrast ridge waveguide made of silicon $(n=3.45)$ on a silicon dioxide $\left(\mathrm{SiO}_{2}, n=1.445\right)$ layer with a width of $500 \mathrm{~nm}$, a height of $240 \mathrm{~nm}$, and deeply etched into the substrate is discussed in the following subsections (see Fig. 5). The holes $(n=1)$ are drilled deeply into the substrate, and the periodicity of the structure is $430 \mathrm{~nm}$. A 3-D FDTD method has been used to simulate the devices with the fundamental TE mode of the waveguide as the incident wave.

Our design process to accomplish high performance of a bandpass filter consists of three main steps. These steps include: 1) constructing both input and output mirrors with small $\kappa$; 2) assembling these two mirrors to compose an AD mirror with its resonant wavelength equal to the center wavelength of the filter and its $\kappa$ at the center wavelength less than 0.051; and 3) obtaining a final bandpass filter design by adjusting the cavity length $d$ between two identical AD mirrors satisfying (7) and (8). Here, the center wavelength of the filter is set at $1554 \mathrm{~nm}$. The steps of the procedures are as follows:

\section{A. PBG Mirror Construction}

It is known that the loss of the PBG mirror could be reduced either by introducing transition-mode-matching structures or by tuning the locations of holes at the interface between the PC mirrors and the cavity as a result of mode matching between the Bloch waves in the PBG mirrors and the guided modes in the cavity [37]-[40]. Here, two different PBG mirrors are discussed: 1) PBG mirror without mode matching section and 2) mirror with mode matching holes at both sides of the mirror, as displayed in the insets of Fig. 6(a) and (b), respectively. The radii of all the holes [see Fig. 6(a)] are chosen to be $150 \mathrm{~nm}$ to obtain a high-reflection zone in the $1550-\mathrm{nm}$ spectrum. The holes with smaller radii on either side of the mirror in Fig. 6(b) act as mode matching features, and their sizes are assigned to reduce the loss of the mirror at the wavelength of $1554 \mathrm{~nm}$. The corresponding simulated reflectance and loss spectra for these two mirrors are graphed in Fig. 6. The mirror in Fig. 6(a) provides a bandgap spectrum ranging from 1100 to $1670 \mathrm{~nm}$, 


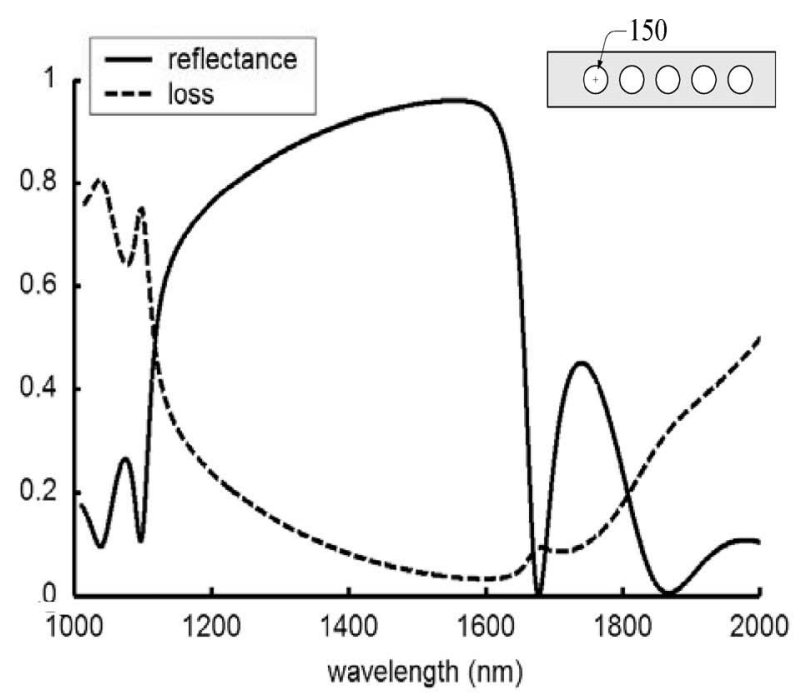

(a)

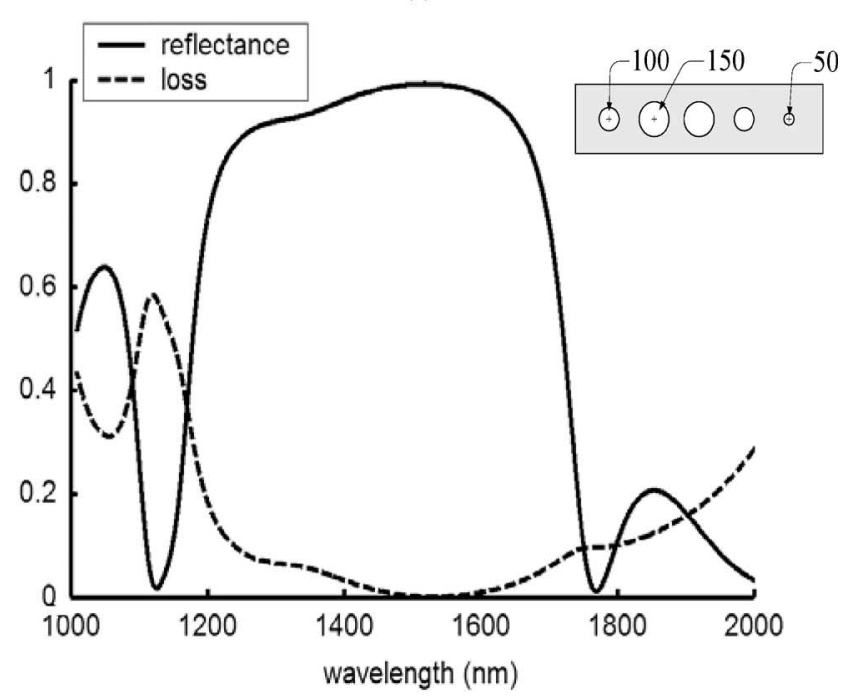

(b)

Fig. 6. Reflectance (solid line) and loss (dashed line) spectra of the PBG mirrors (a) without and (b) with mode-matching section. The inset of each figure is the schematic diagram of the simulated PBG mirrors.

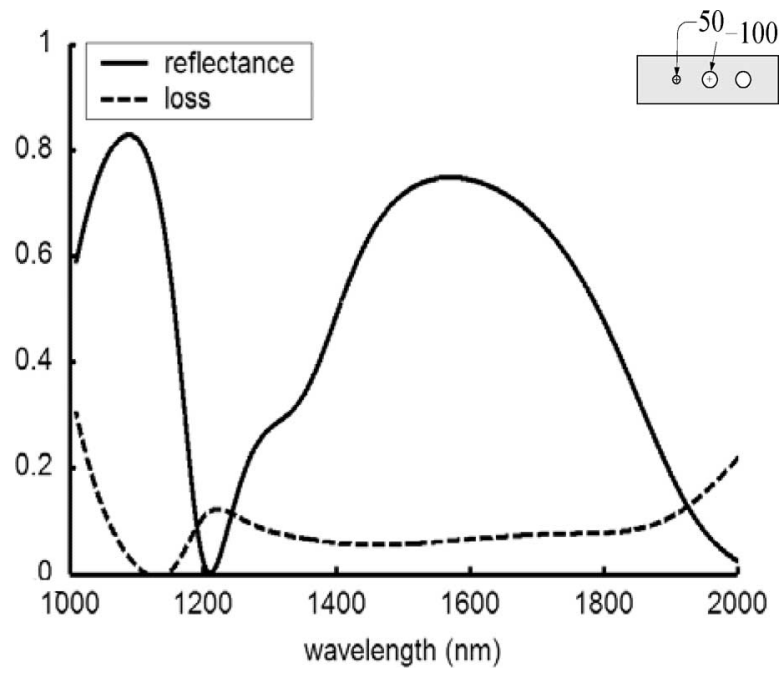

Fig. 7. Reflectance (solid line) and loss (dashed line) spectra of the three-hole PBG mirror and the inset is the schematic diagram of the simulated mirror.

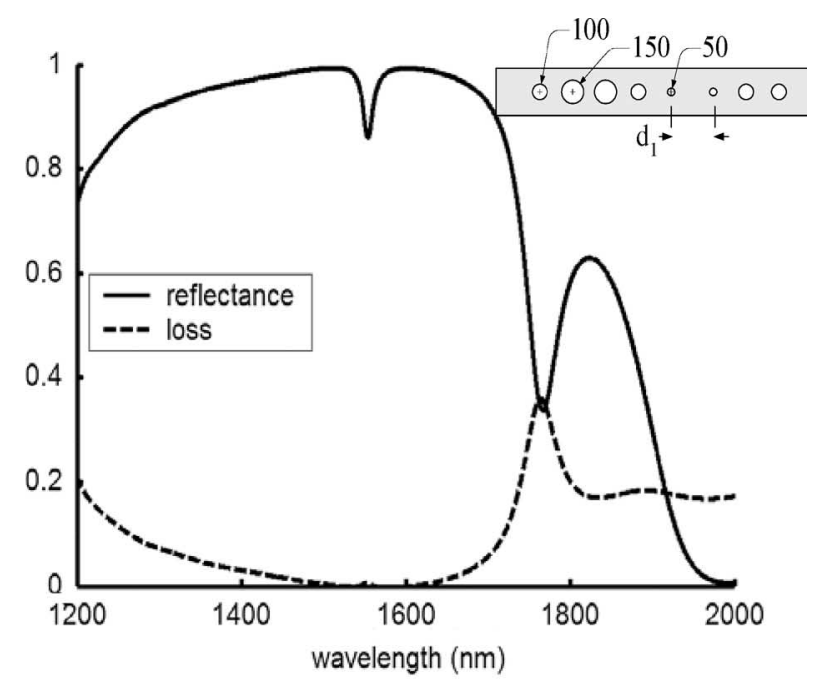

(a)

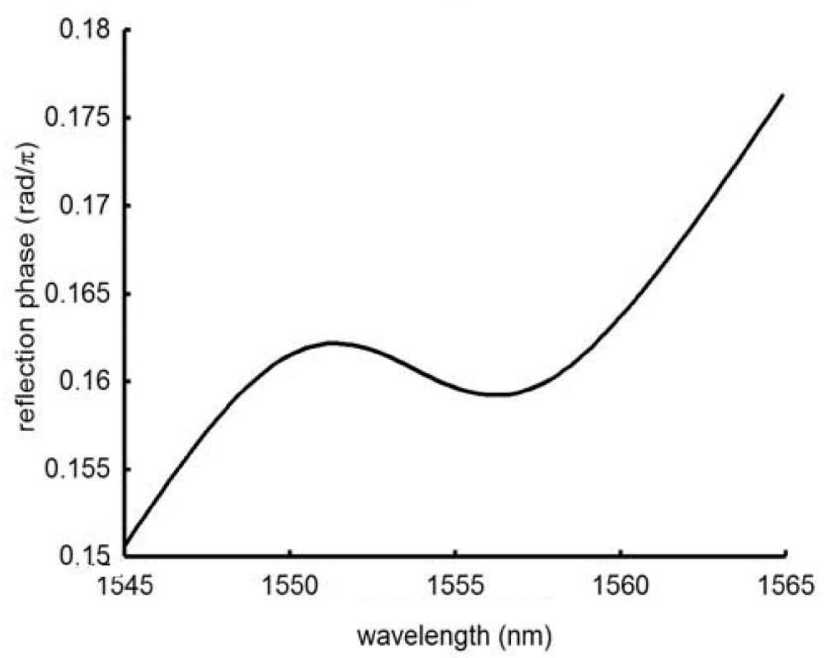

(b)

Fig. 8. (a) Reflectance (solid line) and loss (dashed line) spectra of the $\mathrm{AD}$ mirror with $d_{1}=380 \mathrm{~nm}$. The inset is the schematic structure of the onecavity AD mirror with the cavity's length equal to $d_{1}$. Unit of each label is in nanometers. (b) Calculated reflection phase of this AD mirror.

and that in Fig. 6(b) has a high-reflection zone from 1120 to $1770 \mathrm{~nm}$ with slightly larger reflectance than that of Fig. 6(a). The reflectances at the center wavelength $1554 \mathrm{~nm}$ in Fig. 6(a) and (b) are 0.96 and 0.99 , respectively. By examining their loss spectra, we find that the minimal loss spectra of both designs are occurred in 1400-600 $\mathrm{nm}$ regions, the desired operation spectrum. The loss in Fig. 6(a) at the wavelength of $1554 \mathrm{~nm}$ is roughly 0.036 , and that in Fig. 6(b) is 20 times smaller, i.e., 0.0018 . The calculated $\kappa$ for these mirrors are 0.9 and 0.18 , respectively. Therefore, the modulation of PBG mirror results not only in the reduction of the loss in the mirror, but also in the enhancement of the reflectance of the mirror, thereby realizing much smaller $\kappa$. Here, we use the mirror in Fig. 6(b) as the input mirror for the construction of the AD mirror.

As the input mirror has $R=0.99$ and $\kappa=0.18$, the reflectance of the output mirror needs to be smaller than 0.99, and its $\kappa$ also is chosen to obtain an AD mirror with its $\kappa<0.051$ 


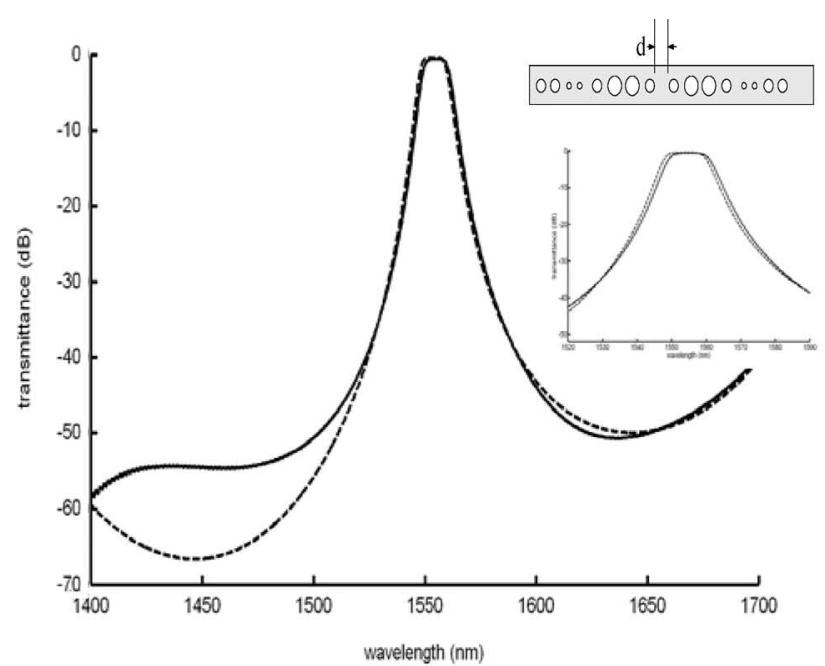

(a)

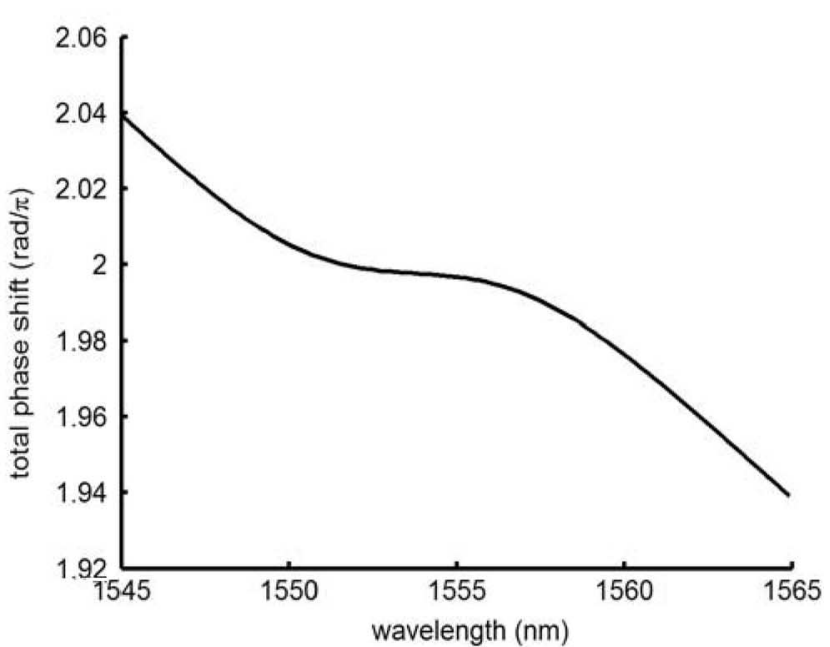

(b)

Fig. 9. (a) Simulated (solid line) and calculated (dashed line) transmission spectra of the FP resonator with the cavity's length $d=367$ nm. The top inset shows the schematic diagram of the simulated PBG waveguide microcavity and the lower inset shows the detailed result around the wavelength between $1520-1590$ nm. (b) Calculated total phase shift inside the cavity $\mathrm{C}$.

at $1554 \mathrm{~nm}$. Let the output mirror be a three-hole PBG mirror with the radii of 50,100, and $100 \mathrm{~nm}$, respectively, as shown in the inset of Fig. 7. The radii of these holes are selected to achieve high reflectance in the $1550-\mathrm{nm}$ spectrum and to provide a mode-matching transition between the input mirror and the input (or output) waveguide. Its reflectance and loss spectra are shown in Fig. 7, and its bandgap spectrum is between 1200 and $2000 \mathrm{~nm}$. At $1554 \mathrm{~nm}$, the reflectance and loss are 0.75 and 0.06 , respectively. Therefore, $\kappa$ of this mirror is 0.24 . The expected reflectance and loss of the composed $\mathrm{AD}$ mirror would be 0.88 and 0.03 .

\section{B. PBG Mirror With the AD Reflection Phase}

At this step, we gather aforementioned mirrors and find the separation distance between these two mirrors defined as $d_{1}$ such that the resonant wavelength is occurred at $1554 \mathrm{~nm}$. The structure of the constructed AD mirror is shown in the inset of Fig. 8(a). In order to obtain the local minimal reflectance at $1554 \mathrm{~nm}, d_{1}$ is chosen to be $380 \mathrm{~nm}$. Fig. 8(a) shows the reflectance and loss spectra of this AD mirror. We observe a dip in the reflectance spectrum occurred at $1554 \mathrm{~nm}$, but a small spike in the loss spectrum at the same wavelength too. The reflectance at $1554 \mathrm{~nm}$ is 0.86 . The loss at $1554 \mathrm{~nm}$ is 0.006 , roughly three times larger than that of the input mirror, but ten times smaller than that of the output mirror. Therefore, the loss of the constructed AD mirror is more affected by the loss of the input mirror than by that of output mirror. In addition, the loss is also five times smaller than that predicted in aforementioned subsection, and the deviation possibly results from negligence of the coupling between radiation modes and Bloch waves in between the separation region. $\kappa$ becomes 0.043 and the maximum transmittance is predicted to be $-0.41 \mathrm{~dB}$ at $1554 \mathrm{~nm}$ by using this AD mirror to construct a waveguide filter.

Fig. 8(b) shows the calculated reflection phase of this AD mirror in the spectrum of $1545-565 \mathrm{~nm}$. We observe an AD region ranging from 1551 to $1556 \mathrm{~nm}$, and a flat passband is expected to obtain in this region as we properly tune the cavity length $d$ of an FP resonator to satisfy the conditions of (7) and (8).

\section{Flat-Top Bandpass Filter Design}

This step of our design procedure is basically to find the length of the cavity $d$ of the FP resonator separating two of the previously devised AD mirrors. The device structure is depicted in the inset of Fig. 9(a). To satisfy the conditions of (7) and (8), $d$ has to be chosen as $367 \mathrm{~nm}$. Fig. 9(a) shows the transmission spectra of this FP resonator with $d=367 \mathrm{~nm}$, both simulated by using FDTD method and calculated by using (6). We see a flat-top passband centered at $1554 \mathrm{~nm}$. The simulated data illustrate the maximal transmission of $-0.55 \mathrm{~dB}$, and FHWH of $13.6 \mathrm{~nm}$. The bandwidth at $-0.5 \mathrm{~dB}$ transmission deduction level is $8.86 \mathrm{~nm}$. Compared to the calculated data, the simulated results are in agreement except in the region of the high-transmission passband with roughly a reduction of 0.03 . In this case, we see that the radiation field slightly mitigates the output field in this passband. Fig. 9(b) shows the total phase shift $\Phi$ as a function of the wavelength, and we observe an approximately constant $(\sim 2 \pi)$ at the wavelength of 1551-1556 nm, corresponding to the flat region of the passband.

\section{Sensitivity of the Designed Filter}

In this subsection, we discuss the possible discrepancy in performance between the ideal design and the fabricated device due to the inaccuracy of the size of the holes in the structure. The radii of all holes in the designed filter are varied by a deviation ratio $\zeta$, and the calculated transmittance of the filters with $\zeta=0.98,1$, and 1.02 are illustrated in Fig. 10. As seen in Fig. 10, the transmittance spectrum shifts to longer wavelengths as $\zeta$ decreases, i.e., the size of the holes become smaller. In addition, the shape of the passband is slightly distorted due to the mismatch of the condition (8). The shift amount of the center 




Fig. 10. Transmission spectrum variations with different deviation ratios $\zeta$ of the radii of the holes: $\zeta=0.98$ (dotted line), $\zeta=1.00$ (solid line), and $\zeta=1.02$ (dashed line).

wavelength and the resonant wavelength of the AD mirrors is roughly $3 \mathrm{~nm}$ as the size of the holes are deviated by $1 \%$. The maximum transmittance at the center wavelength is moderated because of the wavelength-dependent loss of the AD mirrors and the variation of the total phase shift $\Phi$ in the passband so as to dissatisfy the condition (7). In summary, the change in the size of the holes with a constant ratio in a flat-top bandpass waveguide filter structures results in the transmittance spectrum shifting with slight distortion of the passband shape as the deviation ratio is within $\pm 2 \%$.

\section{CONCLUSION}

A new design approach applying thin-film bandpass filter design methodology to implement a multiple-cavity PBG waveguide filter with the characteristics of the flat-top passband and the wide stopband was proposed. The core part of this method is the realization of an $\mathrm{AD}$ mirror with a small value of $\kappa$. For example, here, we demonstrated such a mirror could be constructed by use of a one-cavity structure with the reflectance of the input mirror larger than that of the output mirror and properly choosing the separation distance so as to have a resonance at the center wavelength, where the AD region of reflection phase is located. Subsequently, a multiple-cavity bandpass filter with a nearly rectangular passband is obtained by constructing with two identical AD mirrors separated by a cavity with appropriate length such that the round-trip phase shift inside the cavity satisfies the resonance condition and compensates for the phase dispersion resulting from the AD mirrors as a result of enhancing the transmittance and flattening the transmission band.

To improve the performance of the filters or to reduce the loss of AD mirrors, we can minimize the loss of each PBG mirror for assembling the AD mirror by adding the mode matching sections. In addition, the transmission of the built PBG microcavity filter can be calculated by using the expression of an FP resonator with slight variation of the maximum transmission in the case of $\kappa \ll 1$, and the calculated results are approximately in agreement with the simulation by a 3-D FDTD method. Although the constructed $\mathrm{AD}$ mirror by using one-cavity structure provides higher loss than that of the input PBG mirror, the reduction of reflectance in the concerned spectrum diminishes $\kappa$, thereby increasing the maximum transmission of this type of FP filters. The example of a three-cavity bandpass waveguide filter possesses the maximum transmission of $-0.55 \mathrm{~dB}$ at $1554 \mathrm{~nm}$, FWHM of $13.6 \mathrm{~nm}$, and the width at $-0.5-\mathrm{dB}$ transmission deduction level of $8.86 \mathrm{~nm}$. A better performance could be achieved by optimally minimizing the losses of the PBG mirrors as well as the constructed AD mirrors. These optimized mirrors can be obtained by numerically tuning the sizes and the locations of the holes.

\section{REFERENCES}

[1] V. A. Almeida, C. A. Barrios, R. R. Panepucci, and M. Lipson, "All optical control of light on a silicon chip," Nature, vol. 431, no. 7012, pp. 1081-1084, Oct. 2004.

[2] Q. Xu, B. Schmidt, S. Pradhan, and M. Lipson, "Micrometre-scale silicon electro-optic modulator," Nature, vol. 435, no. 7040, pp. 325-327, May 2005 .

[3] B. Jalali, M. Paniccia, and G. Reed, "Silicon photonics," IEEE Microw. Mag., vol. 7, no. 3, pp. 58-68, Jun. 2006.

[4] R. A. Soref, "Silicon-based optoelectronics," Proc. IEEE, vol. 81, no. 12, pp. 1687-1706, Dec. 1993.

[5] M. Lipson, "Guiding, modulating, and emitting light on siliconchallenges and opportunities," J. Lightw. Technol., vol. 23, no. 12, pp. 4222-4238, Dec. 2005.

[6] B. Jalali, S. Yegnanarayanan, T. Yoon, T. Yoshimoto, I. Rendina, and F. Coppinger, "Advances in silicon-on-insulator optoelectronics," IEEE J. Sel. Topics Quantum Electron., vol. 4, no. 6, pp. 938-947, Nov./Dec. 1998.

[7] D. A. B. Miller, "Optical interconnects to silicon," IEEE J. Sel. Topics Quantum Electron., vol. 6, no. 6, pp. 1312-1317, Nov./Dec. 2000.

[8] J. S. Foresi, P. R. Villeneuve, J. Ferrera, E. R. Thoen, G. Steinmeyer, S. Fan, J. D. Joannopoulos, L. C. Kimerling, H. I. Smith, and E. P. Ippen, "Photonic-bandgap microcavities in optical waveguides," Nature, vol. 390, no. 6656, pp. 143-145, Nov. 1997.

[9] P. Rigby and T. F. Krauss, "Photonics-the $V s$ and $Q$ s of optical microcavities," Nature, vol. 390, no. 6656, pp. 125-125, Nov. 1997.

[10] J. P. Zhang, D. Y. Chu, S. L. Wu, W. G. Bi, R. C. Tiberio, R. M. Joseph, A. Taflove, C. W. Tu, and S. T. Ho, "Nanofabrication of 1-D photonic bandgap structures along photonic wire," IEEE Photon. Technol. Lett., vol. 8, no. 4, pp. 491-493, Apr. 1996.

[11] D. J. Ripin, K. Y. Lim, G. S. Petrich, P. R. Villeneuve, S. H. Fan, E. R. Thoen, J. D. Joannopoulos, E. P. Ippen, and L. A. Kolodziejski, "Onedimensional photonic bandgap microcavities for strong optical confinement in GaAs and GaAs/AlxOy semiconductor waveguides," J. Lightw. Technol., vol. 17, no. 11, pp. 2152-2160, Nov. 1999.

[12] K. Y. Lim, D. J. Ripin, G. S. Petrich, L. A. Kolodziejski, E. P. Ippen, M. Mondol, H. I. Smith, P. R. Villeneuve, S. Fan, and J. D. Joannopoulos, "Photonic band-gap waveguide microcavities: Monorails and air bridges," J. Vacuum Sci. Technol. B, vol. 17, no. 3, pp. 1171-1174, May/Jun. 1999.

[13] J. C. Chen, H. A. Haus, S. Fan, P. R. Villeneuve, and J. D. Joannopoulos, "Optical filters from photonic band gap air bridges," J. Lightw. Technol., vol. 14, no. 11 , pp. 2575-2580, Nov. 1996.

[14] T. F. Krauss, B. Vogele, C. R. Stanley, and R. M. De La Rue, "Waveguide microcavity based on photonic microstructures," IEEE Photon. Technol. Lett., vol. 9, no. 2, pp. 76-178, Feb. 1997.

[15] A. S. Jugessur, P. Pottier, and R. M. De La Rue, "One-dimensional periodic photonic crystal microcavity filters with transition mode-matching features, embedded in ridge waveguides," Electron. Lett., vol. 39, no. 4, pp. 367-369, Feb. 2003.

[16] J. Minowa and Y. Fujii, "Dielectric multilayer thin-film filters for WDM transmission systems," J. Lightw. Technol., vol. 1, no. 1, pp. 116-121, 1983.

[17] P. Baumeister, "Design of a coarse WDM bandpass filter using the Thelen bandpass design method," Opt. Exp., vol. 9, no. 12, pp. 652-657, Dec. 2001 . 
[18] D. Iazikov, C. Greiner, and T. W. Mossberg, "Apodizable integrated filters for coarse WDM and FTTH-type applications," J. Lightw. Technol., vol. 22, no. 5, pp. 1402-1407, May 2004.

[19] T. Kitoh, Y. Inoue, M. Itoh, M. Kotoku, and Y. Hibino, "Low chromatic dispersion flat-top arrayed waveguide grating filter," Electron. Lett., vol. 39, no. 15, pp. 1116-1118, Jul. 2003.

[20] M. K. Smith and C. van Dam, "PHASAR-based WDM-devices: Principles, design, and applications," IEEE J. Sel. Topics Quantum Electron., vol. 2, no. 2, pp. 236-250, Jun. 1996.

[21] K. Madsen and J. H. Zhao, Optical Filter Design and Analysis: A Signal Processing Approach. New York: Wiley, 1999.

[22] M. Oguma, T. Kitoh, K. Jinguji, T. Shibata, A. Himeno, and Y. Hibino, "Passband-width broadening design for WDM filter with lattice-form interleave filter and arrayed-waveguide gratings," IEEE Photon. Technol. Lett., vol. 14, no. 3, pp. 328-330, Mar. 2002.

[23] R. Costa, A. Melloni, and M. Martinelli, "Bandpass resonant filters in photonic-crystal waveguides," IEEE Photon. Technol. Lett., vol. 15, no. 3, pp. 401-403, Mar. 2003.

[24] A. Melloni and M. Martinelli, "Synthesis of direct-coupled-resonators bandpass filters for WDM systems," J. Lightw. Technol, vol. 20, no. 2, pp. 296-303, Feb. 2002.

[25] A. S. Jugessur, P. Pottier, and R. M. De La Rue. (2004, Apr.). Engineering the filter response of photonic crystal microcavity filters. Opt. Exp. [Online]. 12 (7), pp. 1304-1312. Available: http://www.opticsexpress. org/abstract.cfm?URI=OPEX-12-7-1304

[26] R. Zengerle and O. Leminger, "Phase-shifted Bragg-grating filters with improved transmission characteristics," J. Lightw. Technol., vol. 13, no. 12, pp. 2354-2358, Dec. 1995.

[27] Y. V. Troitski, "Dispersion-free, multiple-beam interferometer," Appl. Opt., vol. 34, no. 22, pp. 4717-22, Aug. 1995.

[28] C.-H. Chen, K. Tetz, W. Nakagawa, and Y. Fainman, "Wide-field-of-view GaAs/AlxOy one-dimensional photonic crystal filter," Appl. Opt., vol. 44, no. 8, pp. 1503-1511, Mar. 2005.

[29] S. D. Smith, "Design of multilayer filters by considering two effective interfaces," J. Opt. Soc. Amer., vol. 48, pp. 43-50, 1958.

[30] H. A. Macleod, Thin-Film Optical Filters, 3rd ed. Philadelphia, PA: Institute of Physics Publishing, 2001.

[31] A. Thelen, Design of Optical Interference Coatings. New York: McGraw-Hill, 1989.

[32] G. Sztefka and H. P. Nolting, "Bidirectional eigenmode propagation for large refractive-index step," IEEE Photon. Technol. Lett., vol. 5, no. 5, pp. 554-557, May 1993.

[33] J. Ctyroky, S. Helfert, and R. Pregla, "Analysis of a deep waveguide Bragg grating," Opt. Quantum Electron., vol. 30, no. 5-6, pp. 343-358, 1998.

[34] J. Willems, J. Haes, and R. Baets, "The bidirectional mode expansion method for two-dimensional waveguides: The TM case," Opt. Quantum Electron., vol. 27, no. 10, pp. 995-1007, Oct. 1995.

[35] Y. Y. Troitski, "Dielectric mirrors with the anomalous dispersion of the reflection phase," Opt. Spectrosc., vol. 77, no. 3, pp. 503-506, Sep. 1994.
[36] C.-H. Chen, K. Tetz, and Y. Fainman, "Resonant-cavity-enhanced PIN photodiode with broad quantum efficiency spectrum by use of an anomalous dispersion mirror," Appl. Opt., vol. 44, no. 29, pp. 6131-6140, Oct. 2005.

[37] C. Sauvan, G. Lecamp, P. Lalanne, and J. P. Hugonin, "Modal-reflectivity enhancement by geometry tuning in photonic crystal microcavities," Opt. Exp., vol. 13, no. 1, pp. 245-255, Jan. 2005.

[38] P. Lalanne and J. P. Hugonin, "Bloch-wave engineering for high- $Q$, small$V$ microcavities," IEEE J. Quantum Electron., vol. 39, no. 11, pp. 1430 1438, Nov. 2003.

[39] P. Lalanne, S. Mias, and J. P. Hugonin. (2004, Feb.). Two physical mechanisms for boosting the quality factor to cavity volume ratio of photonic crystal microcavities. Opt. Exp. [Online] . 12 (3), pp. 458-467. Available: http://www.opticsexpress.org/abstract.cfm?URI=OPEX-12-3-458

[40] D. Peyrade, E. Silberstein, P. Lalanne, A. Talneau, and Y. Chen, "Short Bragg mirrors with adiabatic modal conversion," Appl.Phys. Lett., vol. 81, no. 5, pp. 829-831, Jul. 2002.

Chyong-Hua Chen received the B.S. and M.S. degrees in electrical engineering from National Tsing Hua University, Hsinchu, Taiwan, R.O.C., in 1995 and 1997, respectively, and the $\mathrm{Ph}$. D. degree in electrical and computer engineering from the University of California, San Diego, in 2006.

Since February 2006, she has been an Assistant Professor in the Department of Photonics, National Chiao Tung University, Hsinchu. Her current research interests include photonic integrated circuits, photonic crystals, and surface plasmon resonances with the applications of the optical communication systems, the optical sensing systems, and the optical display systems.

Yeshaiahu Fainman (M'93-SM'01-F'03) received the Ph.D. degree from Technion-Israel Institute of Technology, Haifa, Israel, in 1983.

$\mathrm{He}$ is a Professor of electrical and computer engineering at the University of California, San Diego. He has published over 100 manuscripts in refereed journals and over 200 conference presentations and conference proceedings. He has served on several conference program committees and has organized several symposia and workshops. His current research interests include ultrafast information processing with optical nonlinearities and use of femtosecond laser pulses, near-field phenomena in optical nanostructures and nanophotonic devices, quantum communication, multidimensional quantitative imaging, and programmable and multifunctional diffractive and nonlinear optics.

Dr. Fainman is a Fellow of the Optical Society of America. He received the Miriam and Aharon Gutvirt Prize. From 1993 to 2001, he was a Topical Editor of the Journal of the Optical Society of America A and Optical Signal Processing and Imaging Science. 